\title{
Avaliação da Pericardiostomia como método DIAGNÓSTICO E TERAPÊUTICO NO SERVIÇO DE Cirurgia Torácica do Hospital Universitário Gaffrée e Guinle - HUGG.
}

\begin{abstract}
Autores:
Helio Machado Vieira Junior ${ }^{1}$, Aline de Quadros Teixeira $^{1}$, Thiago Scharth Montenegro ${ }^{1}$, Camila Rodrigues de Almeida ${ }^{1}$, Stênio Karlos Alvim Fiorelli ${ }^{1}$, Alfredo Jorge Vasconcelos Duarte ${ }^{1}$, Agostinho Manuel da Silva Ascençáo ${ }^{1}$, Maria Ribeiro Santos Morard ${ }^{1}$, Rossano Kepler Alvim Fiorelli $^{1-2}$

1. Serviço de Cirurgia Torácica - HUGG / Programa de Pós-graduação em Medicina Universidade Federal do Estado do Rio de Janeiro - UNIRIO.

2. Academia Nacional de Medicina
\end{abstract}

\section{Autor correspondente:}

Rossano Kepler Alvim Fiorelli email: fiorellirossano@hotmail.com Tel: (21) 99973-2194

\section{Resumo}

As pericardiopatias constituem um grupo heterogêneo de afecçóes de grande complexidade, ressaltando a importância de sua avaliação como método diagnóstico e terapêutico. Objetivo: Avaliar o perfil epidemiológico e a eficácia das pericardiostomias no diagnóstico etiológico e tratamento dos derrames pericárdicos abordados no Serviço de Cirurgia Torácica do HUGG. Métodos: Quarenta e dois pacientes submetidos à pericardiostomia num período de 20 anos foram agrupados e analisados segundo as variáveis e suas interrelações. Resultados: A idade média dos pacientes foi de 49,36 anos; a relação de mulheres e homens foi de aproximadamente 2:1. O derrame pericárdico foi classificado pelo ecocardiograma como grave em 79,4\% dos casos. A incidência de tamponamento cardíaco foi de 15,3\%. Houve recidiva do derrame em um caso (2,56\%), necessitando nova intervenção cirúrgica. $\mathrm{O}$ exame histopatológico e a análise do líquido pericárdico foram úteis para o estabelecimento do diagnóstico conclusivo. Quanto à etiologia de derrames pericárdicos, houve predomíno de tuberculose, seguida de neoplasia. Conclusóes: As pericardiostomias realizadas foram eficazes como tratamento com baixa taxa de recidiva, e como método diagnóstico em cerca de $90 \%$ dos casos.

Palavras-chave: Derrame pericárdico; pericardiostomia; janela pericárdica. 


\section{Abstract}

Pericardial diseases constitutes a heterogeneous group of disorders of great complexity, highlighting the importance of their evaluation as a diagnostic and therapeutic method.

Objective: To evaluate the epidemiology and effectiveness of pericardiostomies in the etiological diagnosis and treatment of pericardial effusions addressed in the HUGG's Department of Thoracic Surgery.

Methods: Forty-two patients who underwent pericardiostomy over a 20year period were grouped and analyzed according to the variables and their interrelationships.

Results: The average age of the patients was 49.36 years; the ratio of women to men was approximately 2 : 1. Pericardial effusion was classified by echocardiogram as severe in $79.4 \%$ of cases. The incidence of cardiac tamponade was $15.3 \%$. There was a recurrence of the stroke in one case $(2.56 \%)$, requiring a new surgical intervention. Histopathological examination and analysis of pericardial fluid were useful for establishing the conclusive diagnosis. As for the etiology of pericardial effusions, tuberculosis predominated, followed by neoplasia.
Conclusions: Pericardiostomies performed were effective as a treatment with low recurrence rate, and as a diagnostic method in about $90 \%$ of cases.

Keywords: Pericardial effusion; pericardiostomy; pericardial window.

\section{INTRODUÇÃO}

Os derrames pericárdicos são afecções relativamente frequentes na população e consistem num desequilíbrio na produção/ absorção do líquido pericárdico, sobretudo, devido a alteraçôes na permeabilidade das membranas, desencadeadas por diferentes etiologias, tais como: processos inflamatórios e infecciosos, de origem viral, bacteriana, ou fúngica; doenças autoimunes; distúrbios metabólicos; doenças sistêmicas; trauma; radiação; neoplasias; além dos derrames de causa idiopática. ${ }^{1-5}$ Podem ser classificados em: hidropericárdio (transudato), derrame pericárdico "verdadeiro" (exsudato) como o piopericárdio (purulento) ou hemopericárdio (sanguíneo). ${ }^{2,5}$

A presença de líquido eleva a pressão intrapericárdica e depende não somente da quantidade absoluta do mesmo, como também da rapidez de acúmulo e as características físicas do pericárdio. ${ }^{2-5}$ Logo, o quadro clínico do envolvimento do pericárdio é espectral, variando desde a ausência total de sintomas até a presença de choque e parada cardiorrespiratória. ${ }^{6}$ 
Muito se discute sobre a melhor abordagem para os casos de acometimento pericárdico, especialmente se é válida a busca do diagnóstico etiológico. São observadas na literatura descriçóes variáveis de taxas de identificação etiológica de acordo com as técnicas de análise utilizadas: pesquisa direta, citologia, imunofluorescência, testes imunoenzimáticos, reação em cadeia da polimerase, culturas ou biópsias. Com essa finalidade, podem ser realizadas a pericardiocentese ou a pericardiostomia com biópsia. ${ }^{6}$ A escolha do método varia de acordo com a experiência individual, sendo a pericardiostomia com biópsia a mais adequada devido a menor taxa de complicaçôes, recidivas e morbimortalidades, além de maior acurácia diagnóstica e terapêutica ${ }^{7}$, a mais utilizada pelo nosso serviço.

A pericardiostomia, também denominada janela pericárdica, consiste na abertura no pericárdio e drenagem transtorácica de derrames pericárdicos, havendo ainda, a possibilidade de realização de biópsia para melhor investigação diagnóstica. ${ }^{8-10}$ Pode ser realizada por duas vias de acesso: a subxifoidiana e a janela pericárdio-pleural por toracoscopia ou toracotomia anterior, as quais têm bons resultados com alta acurácia. ${ }^{11,12}$ Existe também a possibilidade de ser realizada por via laparoscópica ou transdiafragmática, porém há maior risco de contaminação pelo conteúdo da cavidade peritoneal. ${ }^{7-9,13}$

\section{Casuística e Método}

Estudo observacional, retrospectivo, descritivo de 42 pacientes portadores de derrame pericárdico submetidos à pericardiostomias (subxifoidiana e janela pericárdiopleural) diagnósticas e terapêuticas pelo Serviço de Cirurgia Torácica do HUGG no período de Outubro de 1994 a Março de 2014. Foram agrupados mediante revisão sistemática da documentação redigida em prontuário segundo as variáveis e suas interrelações: sexo, idade, comorbidades, sintomatologia, procedimentos realizados, diagnóstico inicial (clínico, ecocardiográfico e por tomografia computadorizada de tórax), diagnóstico final (histopatológico e análise do líquido pericárdico) e complicaçóes.

Foram incluídos todos os pacientes que foram submetidos a pericardiostomia subxifoidiana e janela pericárdio-pleural. E excluídos os casos de outras intervençôes no pericárdio como pericardiocentese e pericardiectomia isoladas ou ressecçóes de cistos.

\section{Resultados}

A casuística foi composta de 43 casos, realizados em 42 pacientes, pois um dos pacientes realizou o procedimento duas vezes. Do total, 27 (64,2\%) pacientes eram do sexo feminino e $15(35,8 \%)$ do sexo masculino, cujas idades variaram de 19 a 88 
anos (mediana de 50 anos e média de 49, 36 anos).

Dos 43 procedimentos, a pericardiostomia subxifoidiana com biópsia pericárdica foi realizada em 41 (95,3\%) pacientes. Em 2 $(4,7 \%)$ casos foi realizada janela pericárdiopleural com biópsia pericárdica.

Quanto à sintomatologia pré-operatória, dentre os 39 pacientes com dados completos, apenas $1(2,5 \%)$ era assintomático, enquanto $38(97,5 \%)$ apresentavam predominantemente dispneia $(94,8 \%)$, fadiga $(58,9 \%)$, tosse $(46,1 \%)$, perda ponderal (41\%), dor torácica $(35,9 \%)$, palpitação $(35,9 \%)$, e febre $(33,3 \%)$. Outros sintomas como hiporexia $(15,3 \%)$, sudorese $(12,8 \%)$, ortopneia $(12,8 \%)$, náuseas $(7,6 \%)$ e vômitos $(7,6 \%)$ foram menos frequentes (Tabela 1). Foi verificado derrame pleural associado, uni $(25,6 \%)$ ou bilateral $(28,2 \%)$, em $21(53,8 \%)$ pacientes. (Tabela 1$)$

\section{TABela 1}

O derrame pericárdico foi classificado pelo ecocardiograma transtorácico (ETT) como grave (volumoso) em 31 (79,4\%) dos pacientes, dentre os quais $15(38,4 \%)$ apresentavam sinais de restrição diastólica, moderado em 7 (17,9\%) dos pacientes, e leve em 1 $(2,56 \%)$ paciente. O tamponamento cardíaco teve incidência de $15,3 \%$ e foi definido por critérios clínicos e ecocardiográficos.
Dentre as drenagens cirúrgicas realizadas, 27 (64,2\%) ocorreram em caráter de urgência; $25(58,1 \%)$ pacientes foram submetidos à técnica de pericardiostomia subxifoidiana e $2(6,1 \%)$ à janela pericárdiopleural.

Em relação à etiologia dos derrames pericárdicos, 12 pacientes (30,7\%) eram portadores de tuberculose pericárdica, sendo $8(20,5 \%)$ casos diagnosticados por elevada probabilidade e $4(10,2 \%)$ casos confirmados pela biópsia do pericárdio.

No que diz respeito às neoplasias, foram encontrados 12 pacientes (30,7\%), cuja biópsia pericárdica revelou presença de células neoplásicas com pericardite de evolução crônica. Dentre eles, detectou-se 2 casos (16,6\%) de derrames pericárdicos por câncer de mama, 2 (16,6\%) por Linfoma de Hodgkin (LH) subtipo esclerose nodular, $1 \quad(8,4 \%)$ por Sarcoma de Kaposi (SK), 1 (8,4\%) por Linfoma não Hodgkin (LNH), 1 (8,4\%) por câncer de pulmão, $1(8,4 \%)$ por Angiosarcoma, $1(8,4 \%)$ por Timoma invasivo e 1 caso $(8,4 \%)$ de câncer de próstata com metástase pulmonar. Em 2 casos (16,6\%), a biópsia revelou pericardite crônica neoplásica inespecífica.

Foram observados $5(12,8 \%)$ casos de pericardites crônicas de caráter autoimune, sendo 2 (5,1\%) de Lúpus Eritematoso Sistêmico, 1 (2,56\%) de pericardite reumática, 1 (2,56\%) de Doença Mista do Tecido Conjuntivo (DMTC) e 1 (2,56\%) de Esclerose Sistêmica (ES). 
Também foram encontrados $3(7,7 \%)$ pacientes com pericardite aguda bacteriana, cujos microorganismos não foram identificados.

Registrou-se 1 (2,56\%) caso de peri-+ cardite crônica por hipotireoidismo e 4 $(10,2 \%)$ de natureza idiopática.

Em $20(51,2 \%)$ casos foi obtida a característica do fluido drenado durante o ato cirúrgico: transudato em 10 (25,6\%) e exsudato nos outros 10 casos $(25,6 \%)$, sendo $5(12,8 \%)$ hemorrágicos, $3(7,6 \%)$ serofibrinosos e 2 (5,2\%) amarelos-citrino. (Tabela 2)

\section{TABEla 2}

Dos 42 pacientes, 12 (28,5\%) apresentaram complicaçóes intra e pós-operatórias. Houve 1 (2,3\%) óbito operatório (iatrogenia) e 2 (4,6\%) óbitos pós-operatórios não relacionados ao procedimento. No paciente que foi submetido duas vezes ao procedimento, houve na primeira drenagem cirúrgica complicação por síndrome pós-pericardiostomia (SPP) e na segunda, complicação por TVP (2,3\%). Foram constatados 5 (11,9\%) casos de sepse pós-operatória, seguida de óbito e 2 (4,6\%) casos de Edema Agudo de Pulmão pós-extubação.

Analisando-se os resultados anatomopatológicos das biópsias pericárdicas, o diagnóstico conclusivo foi obtido em 21 casos $(48,8 \%)$. Destes, $4(9,3 \%)$ casos corres- ponderam à tuberculose pericárdica, outros $4(9,3 \%)$ à pericardite autoimune, $3(7,0 \%)$ à pericardite de origem bacteriana e os 10 $(23,2 \%)$ restantes à pericardite neoplásica. Nos demais casos $(51,2 \%)$, a etiologia foi definida através da avaliação clínica, laboratorial e/ou radiológica dos derrames pericárdicos e a biópsia permaneceu inconclusiva (Tabela 3).

\section{TABELA 3}

\section{Discussão}

Os derrames pericárdicos sintomáticos ocorrem como resultado de vários processos da doença de base e podem ser tratados com diversos procedimentos cirúrgicos diferentes: pericardiocentese, janela pericárdio-pleural e pericardiostomia subxifoidiana. ${ }^{14-16}$ Cada um destes tratamentos pode ser eficaz, dependendo de fatores clínicos e da história do doente.

O procedimento ideal deve ser de fácil execução, resultando em morbidade e mortalidade mínima, com garantia de drenagem completa e permanente e baixa taxa de recorrência, fornecendo dados histológicos, citológicos e espécimes microbiológicos suficientes para o diagnóstico definitivo. ${ }^{17,18}$

Ainda que a pericardiocentese possa proporcionar um alívio temporário para os pacientes com sintomas e sinais de instabilidade hemodinâmica, não é adequada 
para tratamento definitivo dos derrames pericárdicos, já que, embora seja menos invasiva, está associada a uma maior taxa de morbimortalidade e recidivas e não inclui a visualização ou biópsia do pericárdio, tendo uma eficácia diagnóstica incompleta. ${ }^{14,19}$ Por outro lado, as vantagens potenciais da pericardiostomia são a visualização direta para exploração do pericárdio e cavidade pericárdica, a capacidade de sondar a cavidade para permitir a drenagem completa, a possibilidade de realização de biópsia do pericárdio para análise anatomopatológica e menor tendência a recidivas. ${ }^{14,15,20}$

As pericardiostomias subxifoidianas foram preferencialmente realizadas no nosso estudo, por apresentarem menor morbimortalidade quando comparadas com a outra técnica, a qual é mais utilizada em derrames pericárdicos recidivantes e nos casos de abordagem pleural conjunta. $\mathrm{Na}$ maioria dos pacientes portadores de derrame pleural associado, a descompensação que levou à realização da drenagem cirúrgica foi apenas pericárdica, não justificando, portanto, a execução da janela pericárdio-pleural.

Nesta pesquisa, verificou-se predomínio no sexo feminino $(64,2 \%)$ e a idade dos pacientes variou de 19 a 88 anos. Nos estudos de Becit $^{21}$ e Uchimura $^{7}$ houve um predomínio do sexo masculino $(60 \%$ e $87,5 \%$ respectivamente) e a faixa etária dos pacientes variou de 5 a 78 anos e 15 a 80 anos respectivamente.
A sintomatologia pré-operatória predominante foi dispneia, fadiga, tosse, dor torácica e palpitação, compatível com a literatura. Todos os pacientes com derrame pericárdico sintomático obtiveram alívio imediato após a realização da pericardiostomia.

Derrames pleurais uni ou bilaterais, geralmente, desenvolvem-se em associação ao derrame pericárdico. ${ }^{2-4} \mathrm{Na}$ nossa amostra, isso ocorreu em pouco mais da metade dos pacientes. Todavia, diferente dos dados da literatura, em que a maioria dos pacientes com derrame pericárdico costuma apresentar derrame pleural esquerdo isolado ou maior do que à direita 4,14, a maioria dos pacientes deste estudo apresentou derrame pleural bilateral $(28,2 \%)$, seguido de derrame pleural à esquerda $(15,3 \%)$ e de derrame pleural à direita $(10,3 \%)$.

O principal método diagnóstico inicial para os derrames pericárdicos dos casos estudados foi o ETT. Em 15 casos (38,4\%) dos derrames volumosos, o ETT demonstrou sinais de restrição diastólica, com colabamento de $\mathrm{AD}$ e/ou VD. Nestes pacientes, portanto, havia maior risco ao desenvolvimento de tamponamento cardíaco. A TC de tórax foi utilizada como um método auxiliar, permitindo, em alguns casos, a melhor visualização e quantificação dos derrames. $\mathrm{Na}$ literatura os resultados são similares. ${ }^{21}$

Embora a doença do pericárdio seja uma entidade comum, o tamponamento cardíaco é muitas vezes considerado uma 
característica de apresentação incomum. ${ }^{22-24}$ $\mathrm{Na}$ amostra do estudo, a incidência foi de 15,3\%. No estudo de Becit21 a incidência de tamponamento foi $25 \%$.

No presente estudo, as pericardites tuberculosa e neoplásica foram as mais prevalentes, com frequência de 30,7\% cada uma. Entretanto, no estudo de Becit21 houve uma maior prevalência das pericardites urêmicas $(\mathrm{n}=158 ; 43)$. Vale ressaltar que a prevalência das pericardites é variável nos estudos analisados, porém, em muitos casos foi observada elevada frequência de derrames volumosos cuja biópsia revelou tuberculose ou neoplasia. ${ }^{18,20,21}$

A tuberculose pericárdica apresenta incidência estimada de $1 \%$ a $4 \%$ dos casos de pericardite em todo o mundo. ${ }^{25,26}$ É responsável por cerca de $4 \%$ dos casos de pericardite aguda, 7\% dos casos de tamponamento cardíaco, e, em estudos mais antigos, 6\% dos casos de pericardite constritiva. ${ }^{26,27}$ No entanto, em alguns países subdesenvolvidos, a tuberculose é uma das principais causas de pericardite. ${ }^{26}$ Sua confirmação diagnóstica definitiva ocorre pela identificação do $M$. tuberculosis na cultura do líquido pericárdico ou biópsia pericárdica. $^{25,27,28}$

No atual estudo, foram encontrados 12 pacientes com pericardite e história clínica sugestiva de tuberculose (TB). Em todos estes pacientes, foi realizada biópsia pericárdica por via subxifoidiana, a qual mostrou pericardite tuberculosa em quatro e pericardite crônica inespecífica em oito pacientes. No entanto, nos casos em que a biópsia não demonstrou granulomas, $\mathrm{O}$ diagnóstico de TB pericárdica foi definido por elevada probabilidade através de forte suspeita clínica, identificação de BAAR no escarro, biópsia positiva de gânglio periférico, altos níveis de ADA no líquido pericárdico e/ou imagem radiológica sugestiva. Apesar da importante contribuição da biópsia do pericárdio, a tuberculose pericárdica é ainda uma doença de difícil comprovação laboratorial.

No Brasil, as pericardites tuberculosas ocupam espaço importante entre as efusóes pericárdicas do HIV. Nos últimos anos, a presença deste vírus tem alterado a história natural da tuberculose pericárdica, a qual ocorre mais frequentemente como parte de um processo disseminado, tendo um maior impacto na morbidade $e$ mortalidade. ${ }^{26,28,29} \mathrm{Na}$ ausência do vírus, contudo, a morbidade da TB pericárdica está relacionada essencialmente com a resposta imunológica aos antígenos da TB dentro do pericárdio. $^{6,26,28}$

O acometimento neoplásico do pericárdio é comumente metastático, apresentando-se raramente como neoplasia primária do coração e do pericárdio, e se manifesta com sinais de restrição em número considerável de indivíduos, impondo maior gravidade e contribuindo diretamente para 
a morte em até $83 \%$ dos casos. Ocorre em $1,5 \%$ a $21,6 \%$ das autópsias de pacientes com neoplasia de diferentes tipos. ${ }^{16,30-32} \mathrm{Na}$ série de pacientes estudados, a frequência das neoplasias assemelha-se à frequência encontrada na literatura, em que a maioria é proveniente de metástases de carcinomas de pulmão e mama, além dos linfomas, ao passo que timomas e angiosarcomas são casos raros descritos. ${ }^{16,30,33-35}$

Quanto à biópsia pericárdica, observase, portanto, uma alta positividade na determinação do tipo histológico do tumor a partir da realização da janela pericárdica. Esse dado é similar ao da literatura levantada para o trabalho, o que explica a importância da realização da biópsia para determinar o diagnóstico conclusivo das pericardites neoplásicas. ${ }^{36,37}$

Sabe-se que a pericardite lúpica é a forma mais comum de comprometimento cardiovascular. Apesar de ser frequente, o derrame pericárdico é considerado raro. ${ }^{38,39}$

$\mathrm{O}$ envolvimento cardíaco constitui um dos mais graves eventos orgânicos internos em pacientes com ES, devido a uma elevada taxa de mortalidade associada. É relatado em pelo menos $15 \%$ dos doentes portadores, mas a prevalência é significativamente maior após autópsia. ${ }^{39} \mathrm{Na}$ DMTC, por sua vez, a manifestação clinica cardíaca mais comum é a pericardite, que ocorre entre $10 \%$ a $30 \%$ dos pacientes. O tamponamento cardíaco é raro. ${ }^{18,40,41}$
O derrame pericárdico é uma das manifestaçóes cardíacas mais comumente associadas ao hipotireoidismo, embora sua incidência tenha reduzido após o aumento da frequência de diagnóstico precoce da doença. ${ }^{42}$

Apesar de ser considerado o tipo de pericardite mais frequente, segundo a literatura, ocorrendo geralmente como uma complicação aguda de infecções virais ${ }^{43}$, não houve registro de nenhum caso de pericardite de origem viral tanto no presente estudo como na análise retrospectiva de Becit e colaboradores. Entretanto, a pericardite viral possivelmente foi causa de derrame pericárdio em alguns dos nossos casos de pericardite idiopática, haja vista que os vírus não são rotineiramente cultivados em nosso laboratório de microbiologia.

Quanto às complicaçóes, 12 pacientes apresentaram repercussóes intra ou pósoperatórias. $\mathrm{O}$ único caso intra-operatório ocorreu por iatrogenia durante o ato cirúrgico. O paciente foi inicialmente diagnosticado com derrame pericárdico volumoso, no entanto, durante a cirurgia, foi constatada uma cardiomegalia, sem evidência de derrame e houve um hemopericárdio importante por lesão puntiforme da parede anterior do ventrículo direito, ocasionando PCR e óbito. Posteriormente, o laudo da necropsia evidenciou uma miocardiopatia dilatada idiopática.

No paciente que foi submetido duas vezes à pericardiostomia, houve na primeira 
drenagem cirúrgica complicação por síndrome pós-pericardiostomia (SPP). A SPP é uma complicação relativamente comum que surge após a cirurgia cardíaca; consiste numa reação inflamatória no pericárdio, ocorrendo em um intervalo de dias a semanas após a manipulação cirúrgica. ${ }^{44}$ Neste caso, a segunda drenagem sobre o pericárdio foi realizada após ausência de resposta ao tratamento medicamentoso com anti-inflamatórios, os quais representam a primeira escolha terapêutica para tal síndrome. ${ }^{4}$

Dentre os resultados anatomopatológicos da amostra da atual pesquisa, o diagnóstico conclusivo foi obtido em 21 casos (48,8\%). Assim, a biópsia do pericárdico, mesmo com baixa sensibilidade em detrimento da alta especificidade, apresentou uma porcentagem considerável de resultados positivos, estabelecendo a confirmação do diagnóstico etiológico de quase metade dos derrames pericárdicos do presente estudo, o que possibilitou o tratamento dirigido à causa de base. Dados semelhantes foram encontrados na literatura..$^{20,21,37}$

\section{Conclusão}

A pericardiostomia é uma técnica segura e eficaz, não só para abordar terapeuticamente pacientes com derrame pericárdico, mas também para auxiliar no diagnóstico etiológico, especialmente em pacientes com processos tuberculosos, malignos e autoimunes que invadem o pericárdio.

\section{REFERÊNCIAS BIBLIOGRÁFICAS}

1. Sabiston. Tratado de Cirurgia. A Base Biológica da Prática Cirúrgica Moderna. 18 ed2010.

2. Guyton AC HJ. Tratado de fisiologia médica. 11 ed2006.

3. Lopes AC AN, V. . Tratado de Clínica Médica. 1 ed2006.

4. Kasper Dea. Harrison Medicina Interna. Vol 2. 16 ed2006.

5. Spodick DH. Doenças do Pericárdio. Tratado de Medicina Cardiovascular. Vol 2. 6 ed2003.

6. Hajjar LA, Calderaro D, Yu PC, et al. Manifestaçóes cardiovasculares em pacientes com infecção pelo vírus da imunodeficiência humana. Arquivos Brasileiros de Cardiologia. 2005;85(5):363-377.

7. Uchimura MM, Battiston J, Moreira P, Stahlschmidt CMM, Lubachevski FL. Análise epidemiológica das pericardiotomias realizadas em um hospital universitário de Curitiba. Revista do Colégio Brasileiro de Cirurgiōes. 2010;37(2):092-095.

8. Mantovani M, Espinola JP, Fraga GP. Janela pericárdica transdiafragmática no 
diagnóstico de lesão cardíaca. Revista do Colégio Brasileiro de Cirurgióes. 2006;33(1):29-34.

9. Sellke F, et al. Sabiston \& Spencer, Surgery of the chest. Vol 2. 8 ed2009.

10. Fernandes F, Ianni BM, Arteaga E, Benvenutti L, Mady C. Valor da biópsia de pericárdio no diagnóstico etiológico das pericardiopatias. Arquivos brasileiros de cardiologia. 1998;70(6):393-395.

11. O’Brien PK, Kucharczuk JC, Marshall $\mathrm{MB}$, et al. Comparative study of subxiphoid versus video-thoracoscopic pericardial "window". The Annals of thoracic surgery. 2005;80(6):2013-2019.

12. Petcu C, Droc I. The efficiency of surgical subxiphoid pericardial drainage and percutaneous pericardial drainage in pericardial effusions associated with cardiac tamponade. Chirurgia (Bucur). 2013;108(2):226-233.

13. Watarida S, Shiraishi S, Matsubayashi K, Imura M, Nishi T. Pericardialperitoneal window for chronic exudative pericarditis using a subxiphoidal approach: report of three cases. Surgery today. 2002;32(5):410-413.

14. Maisch B, Ristić AD. Practical aspects of the management of pericardial disease. Heart. 2003;89(9):1096-1103.

15. Mazzucotelli J. Pericardial drainage. The right indication for the right technique. Presse medicale (Paris, France: 1983). 1997;26(22):1051.
16. Cullinane CA, Paz IB, Smith D, Carter N, Grannis Jr FW. Prognostic factors in the surgical management of pericardial effusion in the patient with concurrent malignancy. Chest. 2004;125(4):13281334.

17. Sagristà-Sauleda J, Mercé AS, SolerSoler J. Diagnosis and management of pericardial effusion. World journal of cardiology. 2011;3(5):135.

18. Zanettini MT, Zanettini JO, Zanettini JP. Pericardite: série de 84 casos consecutivos. Arquivos Brasileiros de Cardiologia. 2004;82(4):360-364.

19. Allen KB, Faber LP, Warren WH, Shaar CJ. Pericardial effusion: subxiphoid pericardiostomy versus percutaneous catheter drainage. The Annals of thoracic surgery. 1999;67(2):437-440.

20. Becit N, Özyazicioğlu A, Ceviz M, Karakelleoğlu Ş, Karapolat S, Kocak H. Clinical experience with subxiphoid pericardiostomy in the management of pericardial effusions: a study of 240 cases. Journal of international medical research. 2003;31(4):312-317.

21. Becit N, Ünlü Y, Ceviz M, Koçoğullari C, Kocak H, Gürlertop Y. Subxiphoid pericardiostomy in the management of pericardial effusions: case series analysis of 368 patients. Heart. 2005;91(6): 785-790.

22. Cheng M-F, Tsai C-S, Chiang P-C, Lee H-S. Cardiac tamponade as 
manifestation of advanced thymic carcinoma. Heart \& Lung. 2005;34 (2):136-141.

23. Sagristà-Sauleda J, Mercé J, PermanyerMiralda G, Soler-Soler J. Clinical clues to the causes of large pericardial effusions. The American journal of medicine. 2000;109(2):95-101.

24. Castier MB, Albuquerque EMN, Menezes MEF, Klumb E, Albanesi Fo FM. Cardiac tamponade in systemic lupus erythematosus. Report of four cases. Arquivos brasileiros de cardiologia. 2000;75(5):446-448.

25. Strang J, Nunn A, Johnson D, Casbard A, Gibson D, Girling D. Management of tuberculous constrictive pericarditis and tuberculous pericardial effusion in Transkei: results at 10 years follow-up. Qjm. 2004;97(8):525-535.

26. Syed FF, Ntsekhe M, Gumedze F, Badri M, Mayosi BM. Myopericarditis in tuberculous pericardial effusion: prevalence, predictors and outcome. Heart. 2014;100(2):135-139.

27. Cegielski JP, Devlin BH, Morris AJ, et al. Comparison of PCR, culture, and histopathology for diagnosis of tuberculous pericarditis. Journal of Clinical Microbiology. 1997;35(12):3254-3257.

28. Ntsekhe M, Mayosi BM. Tuberculous pericarditis with and without HIV. Heart failure reviews. 2013;18(3):367-373.
29. Yoon S, Hahn Y-S, Hong JM, Lee O-J, Han H-S. Tuberculous pericarditis presenting as multiple free floating masses in pericardial effusion. Journal of Korean Medical Science. 2012;27(3):325-328.

30. Vaitkus PT, Herrmann HC, LeWinter MM.Treatmentofmalignant pericardial effusion. Jama. 1994;272(1):59-64.

31. Wagner PL, McAleer E, Stillwell E, et al. Pericardial effusions in the cancer population: prognostic factors after pericardial window and the impact of paradoxical hemodynamic instability. The Journal of thoracic and cardiovascular surgery. 2011;141(1):34-38.

32. Han SH, Koh KK, Lee SJ, Seo JG, Choi SJ, Ha SY. Malignant pericardial effusion not diagnosed by pericardial fluid and biopsy: Importance of CT scan. International Journal of Cardiology. 2007;117(1):e53-e55.

33. Corso RB, Kraychete N, Nardeli S, et al. Spontaneous rupture of a right atrial angiosarcoma and cardiac tamponade. Arquivos Brasileiros de Cardiologia. 2003;81(6):611-613.

34. Chang S, Tang H-H, Wang C-C, et al. A standard approach to expose the recurrent laryngeal nerve during endoscopic thyroidectomy. Journal of Laparoendoscopic \& Advanced Surgical Techniques. 2012;22(3): 259-263. 
35. Dong Y, Zhu D, Dong L, Ren L. Primary cardiac angiosarcoma with pulmonary and lumbar metastases presenting as unexplained pericardial effusion in a 41 year-old man. Heart, Lung and Circulation. 2014;23(5):e145-e146.

36. Lestuzzi C. Neoplastic pericardial disease: old and current strategies for diagnosis and management. World journal of cardiology. 2010;2(9):270.

37. de Alba Montero JM, Barrios AS, PérezVelasco JG. Indicaciones de la biopsia pericárdica. Medicine-Programa de Formación Médica Continuada Acreditado. 2013;11(43):2608-2612.

38. Kumar MA, Sathyamurthy I, Jayanthi K. Systemic lupus erythematosus presenting as cardiac tamponade- $\mathrm{a}$ case report. Indian heart journal. 2012;64(1):106-107.

39. Kitchongcharoenying $P$, Foocharoen C, Mahakkanukrauh A, Suwannaroj S, Nanagara R. Pericardial fluid profiles of pericardial effusion in systemic sclerosis patients. Asian Pacific journal of allergy and immunology. 2013;31(4):314.

40. Montera MW, Mesquita ET, Colafranceschi AS, et al. I Brazilian guidelines on myocarditis and pericarditis. Arquivos brasileiros de cardiologia. 2013;100(4):01-36.

41. Maisch B, Seferović PM, Ristić AD, et al. Guidelines on the diagnosis and management of pericardial diseases executive summary: the Task Force on the Diagnosis and Management of Pericardial Diseases of the European Society of Cardiology. European heart journal. 2004;25(7):587-610.

42. Thirone ACP, Danieli RV, Ribeiro VMFC. Derrame pericárdico maciço como manifestação inicial do hipotireoidismo. Arq Bras Endocrinol Metab. 2012;56(6):383-387.

43. Imazio M, Cecchi E, Demichelis B, et al. Indicators of poor prognosis of acute pericarditis. Circulation. 2007;115(21):2739-2744.

44. Kohler I. Estudo dos fatores de risco para o desenvolvimento da síndrome pós-pericardiotomia. 1999.

\section{TABELAS:}

Tabela 1: Frequência dos sintomas

\begin{tabular}{|l|c|c|}
\hline SINTOMA & CASOS (N=39) & $\%$ \\
\hline Dispneia & 37 & $94,8 \%$ \\
\hline Fadiga & 23 & $58,9 \%$ \\
\hline Tosse & 18 & $46,1 \%$ \\
\hline Perda Ponderal & 16 & $41,0 \%$ \\
\hline Dor torácica & 14 & $35,9 \%$ \\
\hline Palpitação & 14 & $35,9 \%$ \\
\hline Febre & 13 & $33,3 \%$ \\
\hline Hiporexia & 6 & $15,3 \%$ \\
\hline Sudorese & 5 & $12,8 \%$ \\
\hline Ortopneia & 5 & $12,8 \%$ \\
\hline Náuseas/Vômitos & 3 & $7,6 \%$ \\
\hline Assintomático & 1 & $2,5 \%$ \\
\hline
\end{tabular}


Tabela 2: Aspecto do líquido pericárdico

\begin{tabular}{|c|c|c|c|}
\multicolumn{2}{|c|}{ LÍQUIDO PERICÁRDICO } & $\begin{array}{c}\text { CASOS } \\
(N=39)\end{array}$ & $\%$ \\
\hline Sem dados & & 19 & $48,7 \%$ \\
\hline Transudato & & 10 & $25,6 \%$ \\
\hline \multirow{3}{*}{ Exsudato } & Hemorrágico & 5 & $12,8 \%$ \\
\cline { 2 - 4 } & Serofibrinoso & 3 & $7,6 \%$ \\
\cline { 2 - 4 } & Exsudato & 2 & $5,2 \%$ \\
\hline
\end{tabular}

Tabela 3: Distribuição dos diagnósticos anatomopatológicos.

\begin{tabular}{|l|c|c|}
\hline \multicolumn{1}{|c|}{ DIAGNÓSTICO } & $\begin{array}{c}\text { CASOS } \\
(\mathrm{n}=43)\end{array}$ & $\%$ \\
\hline Pericardite bacteriana & 3 & $7,0 \%$ \\
\hline Tuberculose pericárdic & 4 & $9,3 \%$ \\
\hline Pericardite neoplásica & 4 & $9,3 \%$ \\
\hline Pericardite autoimune & 10 & $23,3 \%$ \\
\hline Diagnóstico inconclusivo & 22 & $51,1 \%$ \\
\hline
\end{tabular}

